\title{
Emergence and resilience of cooperation in the spatial Prisoner's Dilemma via a reward mechanism
}

\author{
Raúl Jiménez $^{\text {a,d }}$, Haydee Lugo ${ }^{\text {b,d }}$ José A. Cuesta $^{c}$ \\ Angel Sánchez ${ }^{\mathrm{c}, e, *}$ \\ ${ }^{a}$ Departamento de Estadística, Facultad de Ciencias Sociales y Jurídicas, Universidad \\ Carlos III de Madrid, 28903 Getafe, Spain \\ ${ }^{\mathrm{b}}$ Departamento de Economía, Facultad de Ciencias Sociales y Jurídicas, Universidad \\ Carlos III de Madrid, 28903 Getafe, Spain \\ ${ }^{\mathrm{c}}$ Grupo Interdisciplinar de Sistemas Complejos (GISC), Departamento de Matemáticas, \\ Escuela Politécnica Superior, Universidad Carlos III de Madrid, 28911 Leganés, Spain \\ ${ }^{\mathrm{d}}$ Departamento de Cómputo Científico y Estadística, Universidad Simón Bolivar, A. P. \\ 89000, Caracas 1090, Venezuela \\ ${ }^{\mathrm{e}}$ Instituto de Biocomputación y Física de Sistemas Complejos, Universidad de Zaragoza, \\ 50009 Zaragoza, Spain
}

\begin{abstract}
We study the problem of the emergence of cooperation in the spatial Prisoner's Dilemma. The pioneering work by Nowak and May (1992) showed that large initial populations of cooperators can survive and sustain cooperation in a square lattice with imitate-the-best evolutionary dynamics. We revisit this problem in a cost-benefit formulation suitable for a number of biological applications. We show that if a fixed-amount reward is established for cooperators to share, a single cooperator can invade a population of defectors and form structures that are resilient to re-invasion even if the reward mechanism is turned off. We discuss analytically the case of the invasion by a single cooperator and present agent-based simulations for small initial fractions of cooperators. Large cooperation levels, in the sustainability range, are found. In the conclusions we discuss possible applications of this model as well as its connections with other mechanisms proposed to promote the emergence of cooperation.
\end{abstract}

Key words: Emergence of cooperation, Evolutionary game theory

\footnotetext{
$\bar{*}$ Corresponding author.

Email addresses: rjjimene@est-econ.uc3m.es (Raúl Jiménez), haydee.lugo@cantv. net (Haydee Lugo), cuesta@math.uc3m.es (José A. Cuesta), anxo@math.uc3m.es (Angel Sánchez).
} 


\section{Introduction}

The emergence of cooperative behavior among unrelated individuals is one of the most prominent unsolved problems of current research (Pennisi, 2005). While such non-kin cooperation is evident in human societies (Hammerstein, 2003), it is by no means exclusive of them, and can be observed in many different species (Doebeli and Hauert, 2005) down to the level of microorganisms (Velicer, 2003; Wingreen and Levin, 2006). This conundrum can be suitably formulated in terms of evolutionary game theory (Maynard-Smith, 1982; Gintis, 2000; Nowak and Sigmund, 2004; Nowak, 2006) by studying games that are stylized versions of social dilemmas (Kollock, 1998), e.g., situations in which individually reasonable behavior leads to a situation in which everyone is worse off than they might have been otherwise. Paradigmatic examples of these dilemmas are the provision of public goods (Samuelson, 1954), the tragedy of the commons (Hardin, 1968), and the Prisoner's Dilemma (PD) (Axelrod and Hamilton, 1981). The first two of them involve multiple actors, while the latter involves only two actors, this last case being the setting of choice for a majority of models on the evolution of cooperation.

The PD embodies a stringent form of social dilemma, namely a situation in which individuals can benefit from mutual cooperation but they can do even better by exploiting cooperation of others. To be specific, the two players in the PD can adopt either one of two strategies: cooperate (C) or defect (D). Cooperation results in a benefit $b$ to the opposing player, but incurs a cost $c$ to the cooperator (where $b>$ $c>0$ ). Defection has no costs and produces no benefits. Therefore, if the opponent plays $\mathrm{C}$, a player gets the payoff $b-c$ if she also plays $\mathrm{C}$, but she can do even better and get $b$ if she plays $\mathrm{D}$. On the other hand, if the opponent plays $\mathrm{D}$, a player gets the lowest payoff $-c$ if she plays $C$, and it gets 0 if she also defects. In either case, it is better for both players to play $\mathrm{D}$, in spite of the fact that mutual cooperation would yield higher benefits for them, hence the dilemma.

Conflicting situations that can be described by the PD, either at the level of individuals or at the level of populations are ubiquitous. Thus, Turner and Chao (1999) showed that interactions between RNA phages co-infecting bacteria are governed by a PD. Escherichia coli stationary phase GASP mutants in starved cultures are another example of this dilemma (Vulić and Kolter, 2001). A PD also arises when different yeasts compete by switching from respiration to respirofermentation when resources are limited (Frick and Schuster, 2003). Hermaphroditic fish that alternately release sperm and eggs end up involved in a PD with cheaters that release only sperm with less metabolic effort (Dugatkin and Mesterton-Gibbons, 1996). A recent study of cooperative territorial defence in lions (Panthera leo), described the correct ranking structure for a PD (Legge, 1996). And, of course, the PD applies to very many different situations of interactions between human individuals or collectives (Axelrod, 1984; Camerer, 2003). 
In view of its wide applicability, the PD is a suitable context to pose the question of the emergence of cooperation. How do cooperative individuals or populations survive or even thrive in the context of a PD, where defecting is the only evolutionarily stable strategy (Maynard-Smith, 1982; Nowak, 2006)? Several answers to this puzzle have been put forward (Nowak, 2006) among which the most relevant examples are kin selection theory (Hamilton, 1964), reciprocal altruism or direct reciprocity (Trivers, 1971; Axelrod and Hamilton, 1981), indirect reciprocity (Nowak and Sigmund, 1998), emergence of cooperation through punishment (Fehr and Gächter, 2002) or the existence of a spatial or social structure of interactions (Nowak and May, 1992). This last approach has received a great deal of attention in the last decade and has proven a source of important insights into the evolution of cooperation (see Szabó and Fáth (2007) for a recent and comprehensive review). One such insight is the fact that cooperators can outcompete defectors by forming clusters where they help each other. This result, in turn, leaves open the question of the emergence of cooperation in a population with a majority of defectors. Recently, it has been shown (Ohtsuki et al., 2006) that, if the average number of connections in the interaction network is $k$, the condition $b / c>k$ implies that selection favors cooperators invading defectors in the weak selection limit, i.e., when the contribution of the game to the fitness of the individual is very small. However, a general result valid for any intensity of the selection is still lacking.

In this paper, we propose a new mechanism for the emergence of cooperation, which we call shared reward. In this setting, players interact through a standard $\mathrm{PD}$, but in a second stage cooperators receive an additional payoff coming from a resource available only to them and not to defectors. It should be emphasized that similar reward mechanisms may be relevant for a number of specific applications, such as mutualistic situations with selection imposed by hosts rewarding cooperation or punishing less cooperative behavior (see, e.g., Kiers et al. (2003) and references therein). Another context that may be modelled by our approach is team formation in animal societies (Anderson and Franks, 2001), e.g., in cooperative hunting (Packer and Ruttan, 1988). On the other hand, the idea of a shared reward could be implemented in practice as a way to promote cooperation in human groups or, alternatively, may arise from costly signaling prior to the game, when the exchange of cooperative signals among cooperators is free (Skyrms, 2004). As we will see, this scheme makes it possible for a single cooperator to invade a population of defectors. Furthermore, when strategies evolve by unconditional imitation (Nowak and May, 1992), cooperation persists after the additional resource has been exhausted or turned off. We present evidence for these conclusions coming from numerical simulations on a regular network. In the conclusion, we discuss the reason for this surprising result and the relation of our proposal to previous work on evolutionary games on graphs and to public goods games. 


\section{Spatial Prisoner's dilemma with shared reward}

Our model is defined by a two-stage game on a network. In the first stage, players interact with their neighbors and gobtained payoffs as prescribed by the PD game, whose payoff matrix in a cost-benefit context is given by

$$
\begin{aligned}
& \text { C D } \\
& C\left(\begin{array}{cc}
b-c & -c \\
b & 0
\end{array}\right) \text {. }
\end{aligned}
$$

Subsequently, in the second stage of the game, a fixed amount $\rho$ is distributed among all cooperators. It is important to realize at this point that such a two-stage game is only interesting in a population setting: in a two-player game, the second stage would amount to shift the cooperator's payoff by $\rho / 2$ or $\rho$, depending on the opponent's strategy. Then, for $\rho<c$ we would simply have another PD, whereas for $2 c>\rho>c$ we would have the Hawk-Dove or Snowdrift game (Maynard-Smith, 1982), and for $\rho>2 c$ we would have the trivial Harmony game (also called Byproduct Mutualism (Dugatkin et al., 1992; Connor, 1995). In a population setting, the amount received by a cooperator depends on the number of cooperators in the total population and is therefore subject to evolution as the population itself changes.

In order to write down the payoffs for the game after the second stage, we need to introduce some notation. Let us consider a population of $N$ players, each of whom plays the game against $k$ other players. For player $i, 1 \leq i \leq N$, let us denote by $V_{i}$ the number of cooperators among the opponents of $i$, and by $N_{c}$ the total number of cooperators in the population. The payoffs can then be written as follows:

$$
P_{i}= \begin{cases}V_{i} b-k c+\frac{\rho}{N_{c}}, & \text { if } i \text { cooperates } \\ V_{i} b, & \text { if } i \text { defects. }\end{cases}
$$

This mechanism to reward cooperation has been studied by Cuesta et al. (2007) in a game theoretical model of $n$ players with no spatial structure. As stated above, our goal here is to understand whether or not the mechanism of the shared reward can explain the emergence of cooperation in the Prisoner's Dilemma on networks. To address this problem, we will consider below this game in the framework of a spatial setup following the same general lines as Nowak and May (1992) for comparison. We place $N$ individuals on a square lattice with periodic boundary conditions, each of whom cooperates or defects with her neighbors (4, von Neumann neighborhood). We have chosen this neighborhood for the sake of simplicity in the calculation; results for Moore neighborhood [used, e.g., by Nowak and May (1992)] can be obtained in a straightforward manner. After receiveing their payoffs according to (2), all individuals update their strategy synchronously for the next round, by 
imitate-the-best (also called unconditional imitation) dynamics: they look in their neighborhood for players whose payoff is higher than their own. If there is any, the player adopts the strategy that led to the highest payoff among them (randomly chosen in case of a tie). We then repeat the process and let the simulation run until the density of cooperators in the lattice reaches an asymptotic average value or else it becomes 0 or 1 (note that these two states, corresponding to full defection and full cooperation, are absorbing states of the dynamics because there are not mutations).

From the work by Nowak and May (1992), we know that if we begin the simulation with a sufficiently large cooperator density, then the lattice helps sustain the cooperation level by allowing cooperators interacting with cooperators in cluster to survive and avoid invasion by defectors; defectors thrive in the boundaries between cooperator clusters. What we are interested in is in the question as to how the large initial cooperator level required by Nowak and May (1992) may arise; if the initial number of cooperators is small, they cannot form clusters and full defection is finally established. On the other hand, another relevant point is resilience, i.e., the resistance of the cooperator cluster to re-invasion by defectors. In this respect, we note that while the clusters obtained by Nowak and May (1992) did show resilience, their corresponding cooperation level was not large. As we will see below, the mechanism we are proposing will lead to higher cooperation levels with good resilience properties, even for medium costs. To address these issues, we begin by discussing the invasion by a single cooperator placed on the center of the lattice (in fact, on any site, as the periodic boundary conditions make all sites equivalent). This, along with the possible scenarios of invasion by a single defector, will lead to a classification of the different regimes in terms of the cost parameter. Subsequently, we will carry out simulations with a very low initial concentration of cooperators.

\section{Invasion by a single cooperator and resilience of cooperation}

As our strategy update rule is unconditional imitation, the process is fully deterministic, so we can compute analytically the evolution of the process. Thus, for the first cooperator, seeded at time $t=0$, to transform her defector neighbors into new cooperators, it is immediate to see that $\rho>b+4 c$; otherwise, the cooperator is changed into a defector and the evolution ends. If the condition is satisfied, the four neighbors become cooperators, and we have now a rhomb centered on the site of the initial cooperator. In what follows, we discuss the generic situation in the subsequent evolution of the system.

After the initial cooperator has given rise to a rhomb, there will always be four types of players in the system:

- The cooperators in the bulk, that interact with another four cooperators. 
- The cooperators in the boundary, defined as the set of cooperators that have links with defectors. These boundary players have two cooperator neighbors or only one if they are at the corners of the rhomb, but the key point is that they are always connected to a cooperator that interacts only with cooperators.

- The defectors in the boundary, that interact with one (opposite to the corners of the rhomb) or two cooperators.

- The defectors in the bulk, that interact with another four defectors.

For the rhomb to grow two conditions must be met: first of all, the payoff obtained by the boundary cooperators at the corner ( $b-4 c$ plus the reward contribution) has to be larger than that of the boundary defectors with only one cooperator $(b)$; secondly, the payoff obtained by cooperators that have two cooperator neighbors ( $2 b-4 c$ plus the reward contribution) has to be larger than that of the boundary defectors that interact with two cooperators $(2 b)$. If both conditions are verified, defectors are forced to become cooperators by imitation. Therefore, we must have

$$
b-4 c+\frac{\rho}{N_{c}(t)}>b \text { and } 2 b-4 c+\frac{\rho}{N_{c}(t)}>2 b \Longleftrightarrow \frac{\rho}{N_{c}(t)}>4 c .
$$

We thus find that the condition for invasion does not depend on the benefit $b$. In addition, it predicts that invasion proceeds until the rhomb contains too many cooperators so that the condition is not fulfilled anymore. In view of this result, we find it convenient to introduce a parameter to measure the reward in terms of the cost:

$$
\delta \equiv \frac{\rho}{4 c N}
$$

With this notation, the prediction for the invasion by a single cooperator is that it will proceed as long as the fraction of cooperators verifies $N_{c}(t) / N \leq \delta . N_{c}(t)$, the number of cooperators at time $t$, can be easily determined from the recurrence relation for the growing rhomb: in case the cooperators increase, a new boundary layer is added to the rhomb, and we have $N_{c}(t)=N_{c}(t-1)+4 t$, which can be immediately solved (with initial condition $N_{c}(0)=1$ ) to give $N_{c}(t)=2 t^{2}+2 t+$ 1. Inserting this result in the above condition allows to determine the maximum growth time for the cluster, that is $t^{*}=\max \left\{t: 2 t^{2}+2 t+1 \leq \delta N\right\}$, and the fraction of cooperators in the steady state:

$$
\frac{N_{c}\left(t^{*}\right)}{N}
$$

So far, we have seen that when the reward is large enough $(\rho>4 c N)$, full cooperation sets in, whereas for smaller reward, a cooperator cluster grows up to a final size that depends on $\delta$. Interestingly, when $b / 2>c$, the reward mechanism is only needed to establish an initial population of cooperators, i.e., the rhomb is resilient. To show this, notice that boundary cooperators observe the defectors that earn the largest payoff (those with two links to two cooperators) and compare it with the payoff obtained by bulk cooperators; boundary cooperators are linked to both and unconditional imitation will lead them to adopt the strategy of the neighbor with 


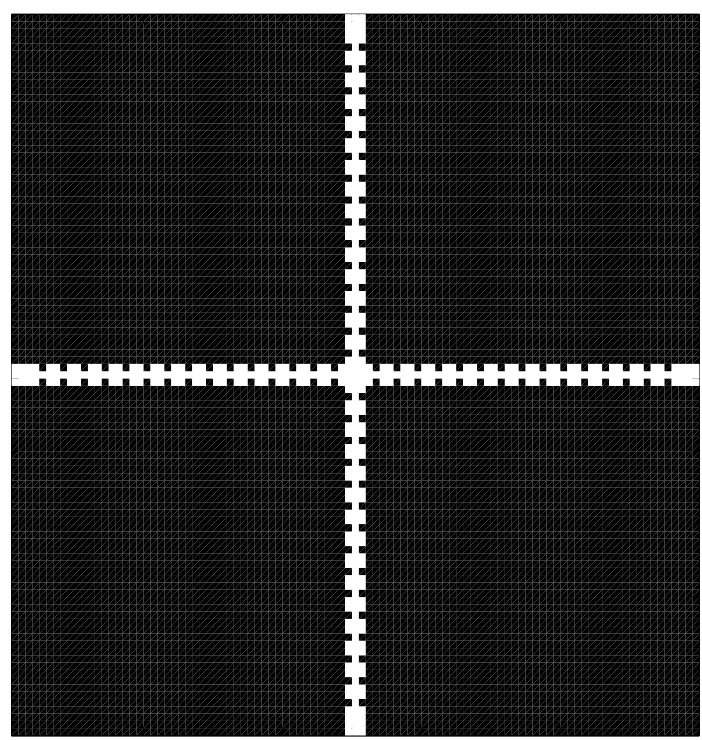

Fig. 1. Final stage of the invasion of a cooperator population by a single defector, for the medium cost case $b / 4<c<b / 2$. Defectors are white, cooperators are black.

the largest payoff. The condition for the cooperators to resist re-invasion is then

$$
4(b-c)>2 b \Longleftrightarrow c<\frac{b}{2}
$$

Indeed, if after a number of time steps we turn off the reward, the rhomb structure arising from the evolutionary process cannot be re-invaded by defectors, as can be seen from Eq. (6). In the opposite case, $c>b / 2$, the reward must be kept at all times to stabilize the cooperator cluster.

In order to study the resilience of clusters of cooperators, we consider the simplest case of invasion by a single defector in the Prisoner's Dilemma (without reward). It can be easily shown that this leads to three different cost regimes (Jiménez et al., 2007):

- Low cost case, $c<b / 4$ : the defector is only able to invade its 4 neighbors, giving rise to a 5 defector rhomb.

- Medium cost case, $b / 4<c<b / 2$ : a structure with the shape of a cross with sawtooth boundaries is formed, implying a finite density of defectors in the final state (cf. Fig. 1).

- High cost case, $c>b / 2$ : the system is fully invaded by the defector, and cooperators go extinct. 


\section{Simulations with an initial concentration of defectors}

After considering the case of the invasion of a defecting population by a single cooperator, we now proceed to a more general situation in which there appear a number of cooperators randomly distributed on the lattice. To this end, we have carried out simulations on square lattices of size $N=100 \times 100$ for different initial numbers of cooperators as a function of the cost parameter (we take $b=1$ for reference) and the reward. A single simulation consists of running the game until a steady state is reached, as shown by the fraction of cooperators becoming approximately constant. Generally speaking, the steady state is reached in some 100 games per player. For every choice of parameters, we compute an average over 100 realizations of the initial distribution of the cooperators. Results are shown in Fig. 2 for low, medium and high costs.

Figure 2 shows a number of remarkable features. To begin with, the case of invation by a single cooperator reproduces the analytical result (5), On the other hand, in all three plots we see that if instead of a single cooperator we have an initial density of cooperators, the resulting level of cooperation is quite higher, particularly when costs are low. Indeed, by looking at panel a), for which $c=0.2(b=1)$, we see that with a $10 \%$ of initial cooperators cooperation sets in even without reward, as observed by Nowak and May (1992). Notwithstanding, a more remarkable result is the fact that with an initial density of cooperators as low as $0.1 \%$ we find large cooperation levels for small rewards, for all values of costs. Clearly, the cooperation level decreases with increasing cost, but even for high costs [panel c), $c=0.7$ ], the cooperation level is significantly higher than the single cooperator one. In this last case, we also observe that the final state becomes practically independent of the density of initial cooperators. Finally, an intriguing result is that in the low cost case, the observed cooperation fraction is not a monotonically increasing function of the reward: As it can be seen from the plot, for moderate and particularly for large initial densities of cooperators, increasing the reward may lead to lower levels

of cooperation. The reason for this phenomenon is that, if the reward increases, the cooperator clusters arising from the cooperator invaders grow larger and overlap. Therefore, clusters with rugged boundaries are formed, allowing for defectors with three cooperators which may then be able to reinvade. Further increments of the reward restore the cooperation levels because then even these special defectors are overrode. The important consequence is that one cannot assume that, for any situation, increasing the reward leads to an increasing of the cooperation, i.e., one has to be careful in designing the reward for each specific application.

The other relevant issue to address in the simulations is the resilience of the attained cooperation levels. Figure 2 summarizes our results in this regard. Both for low and high rewards, we confirm the result for the single cooperator invasion that cooperation disappears if the reward is turned off when the costs are high $(c=$ $0.7>b / 2$ ). For moderate and low costs, the structures arising from the evolution 

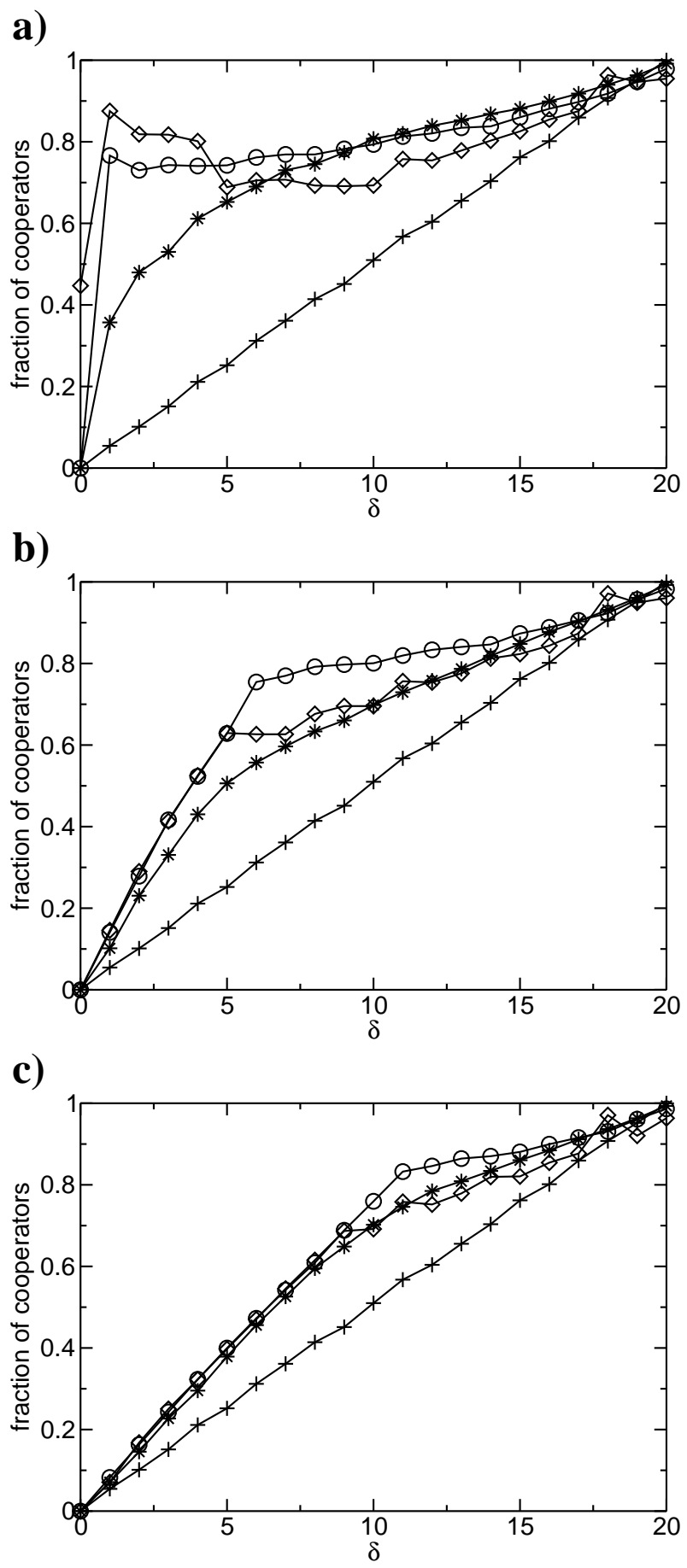

Fig. 2. Average fraction of cooperators in the steady state as a function of the rescaled reward $\delta=\rho / 4 N c$, obtained starting with $1(+), 10(*), 100(\circ)$, and $1000(\diamond)$ initial cooperators. a) low cost, $c=0.2$; b) medium cost, $c=0.4$; c), high cost, $c=0.7$.

with reward do show resilience, at least to some degree. Interestingly, the case of low reward [panel a)] gives rise to extremely robust cooperation levels, whereas higher rewards [panel b)] lead to structures for which cooperation decreases when the reward is absent (medium cost case). This result is connected with the one 
a)

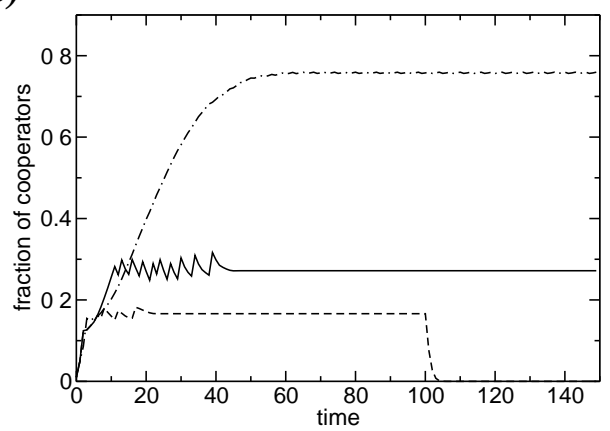

b)

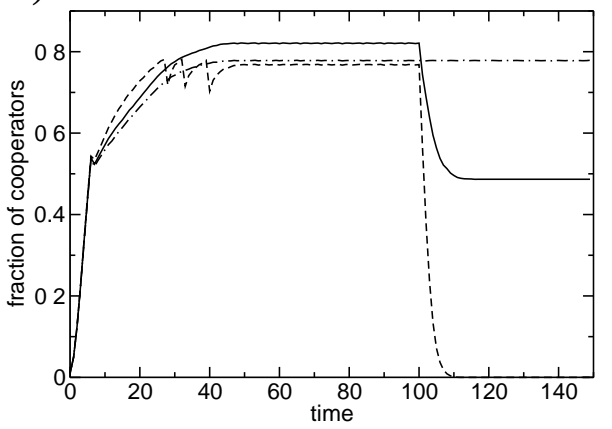

Fig. 3. Time evolution of the fraction of cooperators for the cases of low (dot-dashed line, $c=0.2$ ), medium (solid line, $c=0.4$ ) and high (dashed line, $c=0.7$ ) costs, for simulations starting with 100 initial cooperators (density, 1\%) randomly distributed. Shown are the cases of a) low $(\delta=0.1)$ and $\mathrm{b})$ high $(\delta=0.5)$ rewards. Reward is set in place until $t=100$ and turned off afterwards.

a)

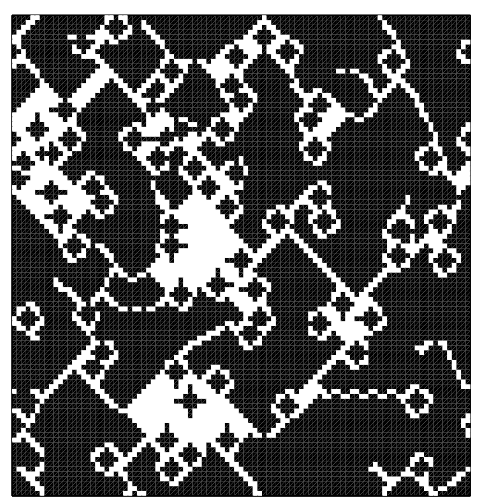

b)

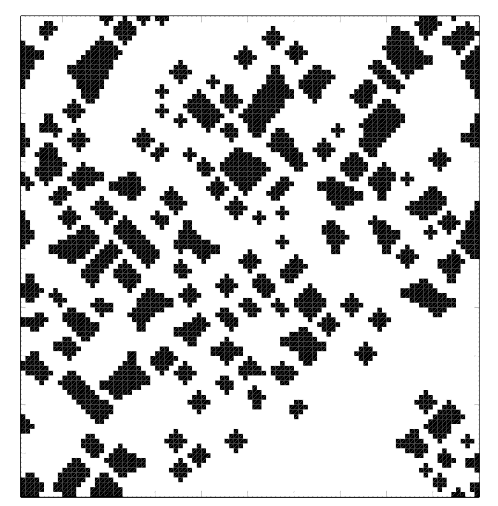

Fig. 4. System snapshots at the stationary state of a single realization of the evolution (before switching off the reward, see Fig. 3) for the low reward case $(\delta=0.1)$. The initial density of cooperators is $1 \%$. a) low cost $(c=0.2)$, b) medium cost $(c=0.4)$. Defectors are white, cooperators are black.

already discussed that the cooperation level may not be monotonics in the reward, and makes it clear that structures originating from a very agressive, high reward policy may be less resilient than those built with low rewards.

Further insight on the cluster structure arising from the invasion process fueled by the reward can be gained from Figs. 4 and 5. Figure 4 shows the stationary structure of the cooperator clusters for the low reward case $(\delta=0.1)$. As we are now considering that the initial configuration contains a $1 \%$ of cooperators randomly distributed, the shapes are irregular, and some rhombs are larger than others because they merge during evolution. In accordance with Fig. 3, in the low cost situation the cooperation level reached is much larger than in the medium cost case. However, both structures are resilient and survive unchanged if the reward is removed. This 
a)

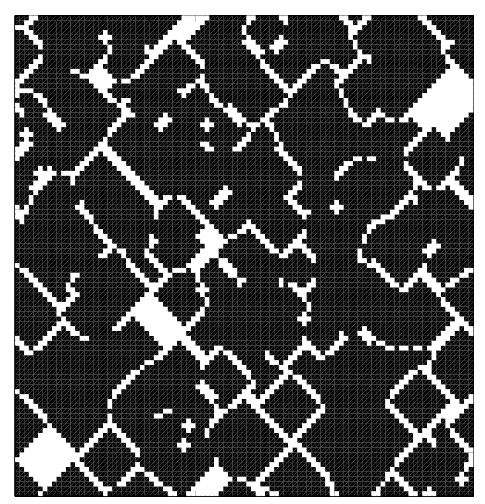

b)

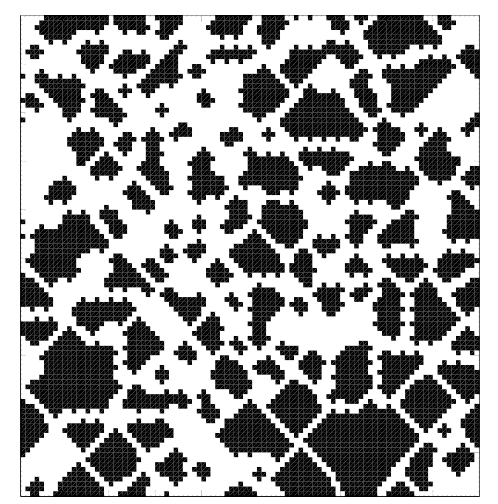

Fig. 5. System snapshots at the stationary state of a single realization of the evolution, a) before and b) after switching off the reward, see Fig. 3) for the high reward case $(\delta=0.5)$ and medium cost $(c=0.4)$. Defectors are white, cooperators are black.

is due to the fact that, as discussed above, in that case defectors can never invade a cooperating population. The final structure for the high cost case is similar to Fig. 4b), but in this case suppression of the reward leads to an immediate invasion by defectors until they occupy the whole system. When the reward is larger, the situation is somewhat different, as can be appreciated from Fig. 5. While for low cost we again obtain resilient structures that are preserved even without reward, in the medium cost regime the patterns change. Panel a) shows the stationary state reached with the reward; when the reward is taken away, the state changes and evolves to the configuration shown in panel b). What is taking place here is that due to the high reward, a cooperation level close to 1 is reached, most of the defectors being isolated or along lines. When the reward is switched off, these defectors are in a position to rip much payoff from their interactions with the cooperators, allowing for a partial reinvasion. Therefore, the final cooperation level has more or less halved. We stress that even then the cooperation level that remains after the suppression of the reward is rather large (about 60\%), another hint of the efficiency of this mechanism to promote cooperation.

\section{Discussion and conclusions}

We have proposed a mechanism that allows a population of cooperators to grow and reach sizeable proportions in the spatial Prisoner's Dilemma in a cost-benefit framework. This mechanism is based in the distribution of a fixed-amount reward among all cooperators at every time step. With this contribution to the payoffs of the standard Prisoner's Dilemma, even a single cooperator is able to invade a fully defecting population. The resulting cooperator fraction is determined by the amount of the reward as compared to the total number of players and to the cost of the in- 
teraction. Furthermore, for low and medium costs $(c<b / 2)$ cooperation is resilient in the sense that if at some time step the reward is suppressed, the cooperator cluster cannot be re-invaded by the defectors. Finally, we have seen that low rewards are capable to induce a very large cooperation level, so the mechanism works even when it changes only a little the payoffs of the Prisoner's dilemma.

The result we have obtained is relevant, in the first place, as a necessary complement of the original work by Nowak and May (1992) within the cost-benefit context. In their work they showed that the spatial structure allowed cooperator clusters to survive and resist invasion by defectors, but they began with a large population of cooperators. Our work provides a putative explanation as to where this population comes from. We note that in the original work by Nowak and May (1992) they observed that the cooperation level decreased with respect to the initial population, so a mechanism leading to the appearance of high cooperator levels is certainly needed. In this regard, we want to stress that the reward mechanism gives rise to structures with very good resilience properties: Simulations without the reward show that starting from a randomly distributed population of cooperators with very large density $(\sim 90 \%)$, the final cooperation level is halved for low costs, and practically disappears for moderate costs.

We stress that, to our knowledge, this is the first time that a mechanism based on a fixed-amount reward to be shared among cooperators is proposed. Notwithstanding, there are other proposals which are somewhat related to ours, most prominent among them being those by Lugo and Jiménez (2006) and Hauert (2006). Lugo and Jiménez (2006) introduce a tax mechanism in which everybody in the population contributes towards a pool that is subsequently distributed among the cooperators. This is different from the present proposal in so far as the contribution from the tax is not a fixed quantity but rather it increases with the average payoff. On the other hand, Hauert (2006) focuses on the effects of nonlinear discounts (or synergistic enhancement) depending on the number of cooperators in the groups of interacting individuals. Although the corresponding game theoretical model, discussed by Hauert et al. (2006) belongs to the same general class of $n$-player games of our shared reward model, the spatial implementation of the two models is very different. Thus, in Hauert (2006), payoffs for a given individual depend on the number of cooperators in her neighborhood, whereas in the present work payoffs depend on the total number of cooperators in the network. On the other hand, our interest is also different, in so far as we are discussing a mechanism to foster the appearance of an initial, sizeable population of cooperators which can later be stable without this additional resource. It is important to stress that with our mechanism a large level of cooperation can be established and (in the appropriate parameter range) stabilized.

We believe that our results may be relevant for a number of experimental situations where the Prisoner's Dilemma has been shown to appear in nature. Thus, the stabilization of mutualistic symbioses by rewards or sanctions as observed in, e.g., 
legume-rhizobium mutualism (Kiers et al., 2003) is related to the mechanism we are proposing here: It is observed that soybeans penalize rhizobia that fail to fix $N_{2}$ in their root nodules. This decreases the defector's payoff which is similar to increasing the cooperator's payoff by a reward. On the other hand, a description of the interaction between different strains of microorganisms [see Crespi (2001); Velicer (2003) and references therein] in terms of this reward mechanism instead of the standard Prisoner's Dilemma may prove more accurate and closer to the actual interaction process. An example could be the evolution of cooperators with reduced sensitivity to defectors in the RNA Phage $\Phi 6$ (Turner and Chao, 1999, 2003). Cooperative foraging is another context where the mechanism of rewarding cooperation may be relevant, ranging from microorganisms such as Myxococcus xanthus (Dworkin, 1996) through beetles (Berryman et al., 1985) to wolves or lions Anderson and Franks (2001). Finally, the question arises as to the validity of such a mechanism to promote cooperation within humans, as individual players can not predict in advance the additional payoff they will obtain from the reward, and therefore it is not clear whether it would have an influence on them or not. Evidences from cooperative hunting in humans (Alvard, 2001; Alvard2, 2003) show that high levels of sharing help sustain cooperative behavior. However, in the human case, contexts where the reward would be more explicitly included in a manner transparent to the players are possible and amenable to experiments. Research along these lines is necessary to assess the possible role of the reward mechanism in specific situations.

\section{Acknowledgments}

This work is partially supported by Ministerio de Educación y Ciencia (Spain) under grants Ingenio-MATHEMATICA, MOSAICO and NAN2004-9087-C03-03 and by Comunidad de Madrid (Spain) under grants SIMUMAT and MOSSNOHO.

\section{References}

Alvard, M. 2001. Mutualistic hunting. In The Early Human Diet: The Role of Meat, Craig Stanford and Henry Bunn, eds., pp. 261-278. Oxford University, New York.

Alvard, M. 2004, Good hunters keep smaller shares of larger pies. Behav. Brain Sci. 27, 560-561.

Anderson, C., Franks, N. R., 2001. Teams in animal societies. Behav. Ecol. 12, 534-540.

Axelrod, R., 1984. The Evolution of Cooperation. Penguin, London.

Axelrod, R., Hamilton, W. D., 1981. The evolution of cooperation. Science 211, 1390-1396. 
Berryman, A. A., Dennis, B., Raffa, K. F., Stenseth, N. C., 1985. Evolution of optima group attack with particular reference to bark beetles (Coleoptera:Scolytidae). Ecology 66, 898-903.

Camerer, C., 2003. Behavioral Game Theory: Experiments in Strategic Interaction. Princeton University Press, NJ.

Connor, R. C., 1995. The benefits of mutualism: a conceptual framework. Biol. Rev. 70, 427-457.

Crespi, B. J., 2001. The evolution of social behavior in microorganisms. Trends Ecol. Evol. 16, 178-183.

Cuesta, J. A., Jiménez, R., Lugo, H., Sánchez A., 2007. Rewarding cooperation in social dilemmas. Working paper 07-52, Departamento de Economía, Universidad Carlos III de Madrid.

Doebeli, M., Hauert, C., 2005. Models of cooperation based on the Prisoner's Dilemma and the Snowdrift game. Ecol. Lett. 8, 748-766.

Dugatkin, L. A., Mesterton-Gibbons, Houston, A. I., 1992. Beyond the prisoner's dilemma: Toward models to discrimate among mechanisms of cooperation in nature. Trends Ecol. Evol. 7, 202-205.

Dugatkin, L. A., Mesterton-Gibbons, M., 1996. Cooperation among unrelated individuals: reciprocal altruism, by-product mutualism and group selection in fishes. BioSystems 37, 19-30.

Dworkin, M., 1996. Recent advances in the social and developmental biology of the Myxobacteria. Microbiol. Rev. 60, 70-102.

Fehr, E., Gächter, S., 2002. Altruistic punishment in humans. Nature 415, 137-140.

Frick, T., Schuster, S., 2003. An example of the prisoner's dilemma in biochemistry. Naturwiss. 90, 327-331.

Gintis, H., 2000. Game Theory Evolving. Princeton University Press, Princeton, NJ.

Hamilton, W. D., 1964. The genetical evolution of social behavior I. J. Theor. Biol. 7, 1-16.

Hammerstein, P. (Ed.), 2003. Genetic and Cultural Evolution of Cooperation. MIT Press, Cambridge, MA.

Hardin, G., 1968. The tragedy of the commons. Science 162, 1243-1248.

Hauert, C., 2006. Spatial effects in social dilemmas. J. Theor. Biol. 240, 627-636.

Hauert, C., Michor, F., Nowak, M. A., Doebeli, M., 2006. Synergy and discounting of cooperation in social dilemmas. J. Theor. Biol. 239, 195-202.

Jiménez, R., Lugo, H., Eguíluz, V., San Miguel, M., 2007. Learning to cooperate. Unpublished manuscript.

Kiers, E. T., Rousseau, R. A., West, S. A., Denison, R. F., 2003. Host sanctions and the legume-rhizobium mutualism. Nature 425, 78-81.

Kollock, P., 1998. Social dilemmas: The anatomy of cooperation. Annu. Rev. Sociol. 24, 183-214.

Legge, S, 1996. Cooperative lions escape the Prisoner's Dilemma. Trends Ecol. Evol. 11, 2-3.

Lugo, H., Jiménez, R., 2006. Incentives to cooperate in network formation. Comp. Econ. 28, 15-27. 
Maynard-Smith, J., 1982. Evolution and the Theory of Games. Cambridge University Press, UK.

Nowak, M. A., 2006. Five rules for the evolution of cooperation. Science 314, 1560-1563.

Nowak, M. A., 2006. Evolutionary Dynamics. Harvard University Press, Cambridge, MA.

Nowak, M. A. May, R. M. May, 1992. Evolutionary games and spatial chaos. Nature 415, 424-426.

Nowak, M. A., Sigmund, K., 1998. Evolution of indirect reciprocity by image scoring. Nature 393, 573-577.

Nowak, M. A., Sigmund, K., 2004. Evolutionary dynamics of biological games. Science 303, 793-799.

Ohtsuki, H., Hauert, C., Lieberman, E., Nowak, M. A., 2006. A simple rule for the evolution of cooperation on graphs and social networks. Nature 441, 502-505.

Packer, C., Ruttan, L., 1988. The evolution of cooperative hunting. Am. Nat. 132, 159-198.

Pennisi, E., 2005. How did cooperative behavior evolve? Science 309, 93.

Samuelson, P. A., 1954. The pure theory of public expenditure. Rev. Econ. Stat. 36, 387-389.

Skyrms, B, 2006. The Stag Hunt and the Evolution of Social Structure. Cambridge University Press, Cambridge, UK.

Szabó, G., Fáth, G., 2007. Evolutionary games on graphs. Phys. Rep., in press.

Trivers, R. L., 1971. The evolution of reciprocal altruism. Q. Rev. Biol. 46, 35-57.

Turner, P. E., Chao, L., 1999. Prisoner's dilemma in an RNA virus. Nature 398, 441-443.

Turner, P. E., Chao, L., 2003. Escape from prisoner's dilemma in RNA phage $\Phi 6$. Am. Sci. 161, 497-505.

Velicer, G. J., 2003. Social strife in the microbial world. Trends in Microbiol. 11, 330-337.

Vulić, M., Kolter, R., 2001. Evolutionary cheating in Escherichia coli stationary phase cultures. Genetics 158, 519-526.

Wingreen, N. S., Levin, S. A., 2006. Cooperation among microorganisms. PLOS Biology 4, 1486-1488. 